\title{
Estrategias de atenuación del lenguaje soez: algunos procedimientos lingüísticos en el doblaje para Hispanoamérica de la película Death Proof
}

\author{
Livia Cristina García Aguiar / Rocío García JimÉnez \\ Universidad de Málaga \\ livia@uma.es / rogarcia@uma.es
}

Recibido: 7 de diciembre de 2012

Aceptado: 27 de febrero de 2013

\section{RESUMEN}

Una de las principales peculiaridades del estilo de los guiones cinematográficos firmados por Quentin Tarantino, como es el caso de Death Proof (2007), consiste en la abundancia de diálogos repletos de coloquialismos, expresiones malsonantes y lenguaje soez, lo cual dota a las conversaciones que mantienen los personajes de la película de un gran realismo.

El doblaje de Death Proof para Hispanoamérica, realizado en español neutro, frente a la versión para España, se caracteriza por presentar una elevada cantidad de casos de estrategias de atenuación del lenguaje soez. El análisis de los distintos procedimientos lingüísticos que llevan a cabo los traductores en la atenuación de las expresiones vulgares constituye el núcleo de esta investigación.

Palabras clave: Estudios Descriptivos, TAV, doblaje, español neutro, atenuación.

Toning down strategies for vulgar language: some linguistic proceedings in the dubbing for Spanish America of the film Death Proof

\begin{abstract}
One of the main characteristics of Quentin Tarantino's films, as happens in Death Proof (2007), is the enormous presence of dialogues full of colloquialisms and vulgar expressions. This peculiarity makes the conversations among the characters of the film very realistic. The dubbing of Death Proof for Spanish America, into standard Spanish, in comparison to that for Spain, shows several cases of toning down strategies of the vulgar language. The analy-


sis of the different linguistic proceedings carried out by translators in order to tone down the vulgar expressions constitutes the center of this research.

Keywords: Translation Descriptive Studies, AVT, dubbing, standard Spanish, toning down.

Sumario: 1. Introducción. 1.1. Marco disciplinar. 1.2. Sobre censura y atenuación. 1.3. Metodología de trabajo. 2. Estrategias de atenuación en Death Proof. 2.1. Introducción. 2.2. Las estrategias de atenuación. 2.3. La atenuación en Death Proof. Análisis del corpus. 3. Conclusiones.

\section{Introducción}

Hasta el momento, la mayoría de los estudios que se realizaban sobre Traducción y Censura se centraban en examinar, principalmente, los aspectos históricos, sociales y culturales que habían influido sobre la traducción ${ }^{1}$. En otras palabras, se analizaba el modo en que determinados factores extralingüísticos, como la censura, incidían en la elaboración del texto meta. Sin embargo, no existe un gran número de trabajos que deje a un lado dichos factores extralingüísticos y se haya propuesto indagar en los procedimientos puramente lingüísticos que se han llevado a cabo para que una traducción afectada por la censura presente los rasgos que la caracterizan como tal. El objetivo del presente trabajo consiste en estudiar y clasificar los diferentes procesos lingüísticos que han derivado del empleo de ciertas estrategias de traducción relacionadas con la censura, como la atenuación, en el caso de la traducción al español de Hispanoamérica de las expresiones malsonantes y los vulgarismos que aparecen en la película Death Proof, traducida al español neutro como A prueba de muerte (Quentin Tarantino, 2007).

\subsection{Marco disciplinar}

El marco en el que se encuadra nuestro artículo es el de los Estudios Descriptivos de Traducción (EDT), cuyas bases quedaron establecidas por James S. Holmes y, sobre todo, por Gideon Toury durante la década de 1980. Fue Holmes quien, en 1980, consideró los Estudios de Traducción como "an empirical discipline" (Holmes, 1980: 71) cuyos objetivos principales eran (1) describir los fenómenos de traducción y las traducciones tal y como estos se manifiestan en el mundo de nuestra experiencia y (2) establecer principios generales a través de los cuales se puedan explicar y predecir dichos fenómenos.

Como cualquier otra disciplina empírica, los Estudios de Traducción se componen de tres ramas que se interrelacionan y son interdependientes: la teórica, la descriptiva y la aplicada. Toury (1980) determinó que la rama descriptiva supone el punto de partida para la elaboración de cualquier teoría traductológica.

\footnotetext{
1 V. ScANdura (2001) y Gómez Castro (2011).
} 
El propio Holmes (1980) estableció que la rama descriptiva podía contar con tres aproximaciones al objeto de estudio:1) la traducción entendida como resultado, 2) la traducción entendida como función, y 3) la traducción entendida como proceso.

Toury se centra en la traducción entendida como resultado, pues considera que las traducciones son "facts of the culture which hosts them" (Toury 1995: 24) y deben ser estudiadas dentro del polisistema en el que son producidas. Toury adopta, por tanto, una perspectiva orientada hacia el polo meta o polo de recepción (targetoriented).

El enfoque escogido por Toury le hace redefinir dos conceptos clave en los Estudios de Traducción, como son el concepto de equivalencia y de norma. Con respecto a la noción de equivalencia, cabe destacar que la visión de Toury abandona los enfoques categóricos y reduccionistas de la lingüística estructural. La equivalencia se transforma en una noción funcional y relacional, de carácter histórico y dinámico existente entre todo original y sus traducciones (Rabadán 1992: 47).

El concepto de norma es entendido por Toury desde un punto de vista sociocultural. Toury (1995: 94) considera el ejercicio de la traducción como un "rol social" llevado a cabo por el traductor. Debido a que esta tiene una dimensión sociocultural, existen una serie de restricciones de distinto grado y tipo que la van a delimitar. En función de la potencialidad de estas restricciones socioculturales, nos vamos a encontrar con un continuum delimitado por dos extremos: en uno se encuentran las reglas generales, que son relativamente absolutas, y en el otro se sitúan los rasgos, caracterizados por ser puramente idiosincráticos, de acuerdo con Toury (1995: 95). Entre estos dos extremos se encuentran las normas, las cuales, según se acerquen a un extremo o al otro, se asemejarán más a las reglas o a los rasgos.

Toury clasificó las normas en tres tipos: la norma inicial (que es la que hará que el traductor se oriente hacia el polo origen o hacia el polo meta), las normas preliminares (las cuales se aplican a aquella información relacionada con la política de traducción que se ha seguido y a la información que determina si la traducción es directa o indirecta) y, por último, las normas operacionales (que son aquellas que dirigen las decisiones que se toman durante el acto de la traducción).

Dentro de las normas operacionales, Toury distingue entre las normas matriciales, que son las que controlan si una traducción está o no completa, es decir, si presenta o no omisiones, y las normas lingüístico-textuales, que son las que estudian el material con el que se formula el texto meta. Debido al enfoque puramente lingüístico que hemos adoptado en el presente trabajo, cabe decir que prestaremos atención, siguiendo la metodología y terminología de Toury, a las normas lingüístico-textuales por las que se ha regido el doblaje que aquí analizamos. No obstante, tampoco debemos olvidar, como se verá más adelante, que la omisión es un fenómeno muy frecuente en nuestro corpus, por lo que las normas matriciales también estarán presentes durante el análisis.

Como acabamos de explicar, nuestro estudio va a estar enmarcado dentro de los Estudios Descriptivos de Traducción, pues pretendemos describir las decisiones que se han tomado en una traducción orientada hacia el polo meta y entendida como producto. Dentro de ello, haremos especial hincapié en las normas operacionales (divididas en matriciales y lingüístico-textuales) definidas por Toury. 


\subsection{Sobre censura y atenuación}

No debemos ignorar los elementos censores que han influido en el proceso de toma de decisiones durante el acto de la traducción. La censura se produce, de acuerdo con Franco Aixelá y Abio Villarig (2009: 111), cuando "el original presenta visiones del mundo que desbordan la tolerancia del polo de recepción". La consiguiente censura, según estos autores, se presentará en forma de atenuación, donde la omisión es una estrategia bastante común.

Los motivos por los cuales se puede producir la censura están relacionados, de acuerdo con Abellán (1980), con la moral sexual, la política, el lenguaje inadecuado y la religión ${ }^{2}$. Scandura (2004) ahonda en los criterios de censura que se aplican en traducción audiovisual. Por una parte, menciona los motivos políticos, según los cuales los gobiernos deciden proteger o controlar a sus ciudadanos impidiéndoles tener contacto con otras culturas o maneras de pensar. Otra de las causas que pueden motivar la censura está relacionada con lo que se considera políticamente correcto. En tercer lugar, la religión actúa como agente censor en una gran cantidad de productos audiovisuales. Finalmente, Scandura (2004: 126) trata el concepto de autocensura del traductor, que se produce cuando el propio traductor no ejerce la censura por motivos externos o ajenos a su voluntad, sino que previendo que su traducción pueda ser modificada o mutilada, decide aplicar sus propios criterios de censura ${ }^{3}$. Las estrategias de atenuación que se han aplicado en el doblaje analizado responderían a un deseo de autocensura por parte del traductor, ya que no tenemos constancia de que se haya ejercido una intervención censora sobre su trabajo.

Debido a que, como hemos venido repitiendo a lo largo de esta introducción, nuestro enfoque es descriptivo, no caeremos en el error de considerar estos fenómenos de atenuación como "no traducciones", "adaptaciones" o incluso "malas traducciones". Compartimos la opinión de Franco Aixelá y Abio Villarig (2009: 113) de que:

Resulta evidente e incluso inevitable que el investigador que desee explicar los mecanismos traductores debe contemplar y analizar como traducción todo mecanismo imaginable (por no hablar de los realmente aplicados) que use un traductor, entre otras cosas porque la renuncia a hacer uso de una estrategia determinada puede ser tan reveladora como el uso de otra opción. [...] La traducción no es inmanentemente represora ni conservadora ni progresista. Por el contrario, constituye un ejercicio de comunicación que debe adaptarse a unas circunstancias comunicativas determinadas que vienen dictadas por la visión del mundo de los responsables de la traducción (con el iniciador y el traductor en primer lugar) como agentes que tienden a ser muy representativos de los valores establecidos en el polo receptor, también en lo ideológico.

\footnotetext{
2 Véanse también Toledano Buendía (2003) y Al-Quinai (2005).

3 Véase también Miquel CoRTÉs (2005).
} 


\subsection{Metodología de trabajo}

Nuestro objetivo es definir y clasificar, desde una perspectiva puramente descriptiva y atendiendo a las normas operacionales de Toury, los diferentes procedimientos lingüísticos (frutos del empleo de ciertas estrategias de traducción donde la censura es un factor importante, como ocurre con la atenuación) que se observan en nuestro corpus. Dicho corpus está compuesto por 69 ejemplos extraídos del doblaje al español de Hispanoamérica (realizado en México, en el estudio CBAudio) de la película Death Proof (2007). El doblaje se ha presentado en la variante del español conocida como español neutro. Los 69 ejemplos son un compendio de las expresiones malsonantes y vulgarismos que aparecen en el film de Quentin Tarantino.

Es necesario señalar que para el análisis de nuestro corpus no hemos tenido en cuenta el doblaje como posible condicionante de las estrategias de traducción empleadas, es decir, el doblaje no ha sido considerado como un elemento extralingüístico que pudiera influir en la elección de las estrategias de atenuación examinadas en el presente artículo.

Existen otros estudios dedicados al análisis de las características que presentan los doblajes al español neutro. Pons (2011), por ejemplo, realiza un análisis comparativo de los doblajes al español de España y al español neutro de la serie televisiva Friends. En un trabajo anterior (García Aguiar y García Jiménez, 2012) examinamos las diferencias que presentaban los doblajes al español neutro y al español de España de la película de la que hemos extraído nuestro corpus, esto es, Death Proof. Los resultados del análisis demostraron que en la versión doblada al español neutro se habían atenuado las expresiones malsonantes y vulgares, en comparación con la versión en español de España. Tales resultados propiciaron el presente estudio (que toma como corpus exclusivamente la versión del doblaje para Hispanoamérica, realizado al español neutro), centrado esta vez en la identificación y descripción de las diferentes estrategias de atenuación empleadas.

Como se verá más adelante, las estrategias de atenuación que hemos observado en nuestro corpus han sido la omisión o elipsis, el circunloquio y el eufemismo (dentro del cual también se ha incluido el trasplante).

\section{Estrategias de atenuación en Death Proof}

\subsection{Introducción}

Antes de entrar de lleno en el análisis lingüístico de las diferentes estrategias de atenuación empleadas en el doblaje al español neutro de la película Death Proof, realizaremos una breve introducción sobre la película y los motivos que nos han llevado a elegirla como base de estudio para nuestra investigación. Asimismo, ofreceremos una definición de la variedad lingüística a la que ha sido doblada la película, esto es, como acabamos de mencionar, el español neutro. De este modo, y basándonos en publicaciones anteriores (García Aguiar y García Jiménez 2010, 2011 y 2012), pretendemos justificar los motivos de elección de nuestro corpus, así como describir las características generales del español neutro. 
Death Proof (2007) es la quinta película del director y escritor estadounidense Quentin Tarantino, quien quiso homenajear con este trabajo las películas de coches de los años setenta. El film narra las macabras persecuciones que el ex-especialista de cine Mike (papel que interpreta Kurt Russell) lleva a cabo con dos grupos de chicas en Austin, Texas, y Lebanon, Tennessee. El objetivo de las persecuciones de Mike no es otro que el de asesinar a las chicas, y para ello utilizará su coche diseñado a prueba de muerte (death proof en inglés). La película constituye el segundo pase de una sesión doble titulada Grindhouse (nombre por el que eran conocidos los cines de barrio norteamericanos en los que se proyectaban sesiones dobles). La sesión doble comienza con la proyección de Planet Terror, escrita y dirigida por Robert Rodríguez (2007).

La manera de escribir de Quentin Tarantino queda especialmente patente en Death Proof, caracterizada por la presencia de una gran cantidad de diálogos, todos ellos plagados de expresiones coloquiales y vulgares. Los guiones de Tarantino se distinguen, precisamente, por esa abundancia de palabras malsonantes, alusiones sexuales y coloquialismos. Por consiguiente, a la hora de doblar o subtitular cualquiera de sus películas (como sucede, en nuestro caso, con Death Proof), se debe tener en cuenta este tipo de lenguaje, que debe ser respetado si se quiere mantener una de las esencias del film.

En anteriores investigaciones (García Aguiar y García Jiménez 2012), hemos podido comprobar cómo la traducción al español neutro, correspondiente a la versión del doblaje para Hispanoamérica, en contraposición con el doblaje para la Península, se caracterizaba por la presencia de una elevada cantidad de casos de estrategias de atenuación de este lenguaje soez. En otras palabras, a través de nuestros análisis observamos que el doblaje para Hispanoamérica tendía a moderar el tono general del lenguaje más vulgar mediante el empleo de eufemismos y omisiones.

Esto nos podría llevar a pensar que la versión hispanoamericana se había visto afectada por la censura (entendida en su sentido más amplio y clásico). No obstante, el hecho de que dicha versión se presentara en español neutro nos hizo cambiar de parecer. El español neutro ${ }^{4}$ es una variedad lingüística estandarizada empleada, principalmente, en la traducción de productos audiovisuales (doblajes, subtitulado, etc.) con la intención de que la misma sea válida para todo el mercado hispanohablante. Por tanto, el español neutro se distingue por ser una variedad lingüística libre de modismos, localismos, etc. y entendible por cualquier hispanohablante ${ }^{5}$. Consecuentemente, no fue de extrañar que la versión hispanoamericana se desprendiese de todos los coloquialismos, modismos o expresiones malsonantes para que fuese entendida por cualquier hablante de Hispanoamérica. Esto conllevó la pérdida de uno de los rasgos lingüísticos más significativos de Death Proof, pero al mismo tiempo, supone una rica fuente de información lingüística sobre las diferentes estrategias traductoras de atenuación (como son los eufemismos, la omisión o la reducción del contenido, entre otros) que intentaremos definir y clasificar en este artículo.

\footnotetext{
${ }^{4}$ Cabe destacar que esta variedad lingüística cuenta con otras denominaciones, como español internacional, español común, castellano general o español estándar. En nuestros trabajos nos hemos decantado por el término español neutro.

${ }^{5}$ Para mayor información sobre la variedad del español neutro, véase RAMíREZ LuENGO (2011), PAJARES GIMÉNEZ (2011) y PONS (2011: 62-63).
} 


\subsection{Las estrategias de atenuación}

Siguiendo las palabras de Franco Aixelá y Abio Villarig (2009: 109), consideramos la traducción como "una actividad compleja e históricamente determinada que tiende claramente a ajustarse a las poéticas y tablas de valores vigentes en una sociedad dada, ofreciendo soluciones netamente distintas a los mismos problemas de traducción a partir de las necesidades vigentes en la sociedad de recepción”. Uno de estos problemas se plantea en la traducción de elementos ideológicamente comprometidos. En estos casos, "o bien el original presenta visiones del mundo que desbordan la tolerancia del polo de recepción, con la consiguiente censura (típicamente en forma de atenuación mediante la estrategia de omisión) o bien el original se amolda a la visión del mundo que se encuentra dispuesto a tolerar el polo receptor" (Franco Aixelá y Abio Villario 2009: 111-112).

En el caso del doblaje que nos ocupa, el traductor se sirve de distintas estrategias a la hora de traducir términos sexuales, en la alusión a la excreción y a otros fenómenos corporales, o en la traducción de insultos, palabrotas en general (lo que se conoce como disfemismo). Uno de los procedimientos mediante el cual el traductor adapta este tipo de lenguaje a la realidad del nuevo contexto sociocultural y lingüístico al que ha de ser traducido es el de la atenuación. La definición que la Real Academia proporciona en la última edición de su diccionario para este término es la de 'Figura que consiste en no expresar todo lo que se quiere dar a entender, sin que por esto deje de ser bien comprendida la intención de quien habla. Se usa generalmente negando lo contrario de aquello que se quiere afirmar' (s.v.). No obstante, desde el punto de vista traductológico se entendería, más concretamente, como una reducción de la explicitud o intensidad, incluyendo el procedimiento formal de omisión (Franco Aixelá y Abio Villarig 2009: 126).

Dentro de este procedimiento general de interdicción lingüística ${ }^{6}$, el traductor recurre a distintas estrategias para atenuar la traducción del texto origen.

Por una parte, puede optar por la omisión o elipsis, consistente en la supresión en el texto meta del término soez o vulgar presente en el texto origen. Junto con esta estrategia, existe una serie de procedimientos gramaticales que sirven para tal fin, como los circunloquios o el cambio en el tiempo y modo verbal ${ }^{7}$. Entendemos el circunloquio como el procedimiento gramatical que consiste en la traducción de la expresión que se quiere evitar mediante un rodeo que alude a la misma referencia, jugando con la complicidad del interlocutor o espectador en este caso. Este procedimiento es el que se emplea cuando en lugar de traducir "get laid" por "echar un polvo", por ejemplo, se prefiere la expresión "pasar una buena noche", o, dentro del propio español, cuando decimos "interrupción voluntaria del embarazo" en lugar del término "aborto". Además, los cambios en el tiempo y modo verbal pueden contribuir a hacer el texto más o menos formal o cortés, como sucede cuando empleamos las formas del condicional en las peticiones (" ¿me podrías pasar la sal?” en lugar de “¿puedes pasarme la sal?” o "pásame la sal”).

${ }^{6}$ Que podemos definir como la coacción que prescribe no hablar de una cosa o, si es necesario hacerlo, indicarla con términos que la sugieran (véase SENABRE 1971: 175).

7 Véase Montes GiRALdo (2003). 
Junto a estas estrategias gramaticales de atenuación, encontramos procedimientos léxicos, también conocidos como eufemismos. El eufemismo (consistente en la sustitución del tabú por un equivalente léxico más indeterminado, menos marcado sociolingüísticamente, más neutral) se produce cuando el interlocutor necesita hacer una referencia cuya actualización le resulta inoportuna y desagradable. Así, "la tensión eufemística nace de la tensión en que se encuentra el hablante cuando debe actualizar una referencia que, por otra parte, desea evitar. En estos casos lo que busca el hablante es suavizar su responsabilidad en lo que interprete el receptor" (Del Teso 1988: 198). Generalmente, el eufemismo recurre a la indeterminación semántica como medio de expresión, de manera que podemos decir que, por lo general, los eufemismos serán signos más indeterminados que los tabúes a los que sustituyen. Eufemismos serían, por ejemplo, "bebido" por "borracho", "mayor" por "anciano" o "acostarse" como traducción de "fuck". Dentro del procedimiento léxico del eufemismo incluimos también el trasplante, con el que aludimos al mecanismo eufemístico de sustitución del disfemismo por un término científico o especializado, como sucedería cuando empleamos "país subdesarrollado" en lugar de "país pobre".

En resumidas cuentas, las estrategias de atenuación que consideraremos en este análisis son las de omisión o elipsis, circunloquio y eufemismo, y, dentro de este último, el trasplante.

Por otra parte, podemos matizar que la necesidad de emplear estrategias de atenuación, tanto en las traducciones como en la lengua general, no viene motivada únicamente por la realidad a la que hemos de referirnos, sino que la situación de discurso representa también un papel muy importante. De manera que determinados elementos son tabú únicamente en situaciones de formalidad, mientras que no lo son en un discurso familiar o coloquial. Así, por ejemplo, en el tipo especial de discurso del lenguaje audiovisual quizás encontremos un mayor grado de formalidad en el que el espectador esperaría encontrar eufemismos ${ }^{8}$. Esta es una de las posibles explicaciones que justificarían el empleo de estrategias de atenuación en el doblaje de la película que es objeto de nuestro estudio. Confirmando esta hipótesis, Scandura (2004: 131-132) recoge los resultados de una encuesta llevada a cabo por la cadena de salas de cine Yelmo Cineplex de Buenos Aires en 2002 y según la cual los espectadores aprueban la aplicación de dichas estrategias atenuadoras en los doblajes por lo que consideran una cuestión "de respeto y educación".

Por otra parte, la interdicción lingüística o atenuación se caracteriza por su inestabilidad. Es decir, dado que se trata de un fenómeno de origen social, la necesidad de aplicar estrategias de atenuación (y el empleo de un tipo u otro de estrategia) dependerá de la sociedad a la que se dirija el texto. Incluso dentro de una misma sociedad también encontramos una gran diversidad. Como apunta Ricardo Senabre, "Estas divergencias [entre hablantes coetáneos] se centran en los objetos sometidos a interdicción, por una parte, y en la elección de sustitutos, por otra" (Senabre 1971: 178). Esto explicaría las diferencias que encontramos en los distintos doblajes de la misma película para España y para Hispanoamérica9 .

8 V. Del TESO (1988: 200-201).

9 V. García Aguiar y García JimÉNez (2012). 


\subsection{La atenuación en Death Proof. Análisis del corpus}

A continuación expondremos en forma de cuadro los resultados del análisis de todas aquellas estructuras en las que se han empleado estrategias de atenuación en el doblaje de Death Proof para Hispanoamérica. Dividiremos los ejemplos atendiendo al tipo de procedimiento empleado para la atenuación: omisión, circunloquio, eufemismo y trasplante.

\begin{tabular}{|c|c|c|}
\hline Versión original & Doblaje para Hispanoamérica & $\begin{array}{l}\text { Estrategia de atenuación: } \\
\text { OMISIÓN }\end{array}$ \\
\hline $\begin{array}{l}\text { Well, you know, not much. } \\
\text { We just fucking met each } \\
\text { other }\end{array}$ & $\begin{array}{l}\text { Pues eso, nos acabamos de conocer. } \mathrm{Si} \\
\text { les das lo que quieren la primera } \\
\text { noche, nunca te respetarán }\end{array}$ & $\begin{array}{l}\text { We just fucking met }<\text { nos acabamos } \\
\text { de conocer } \varnothing\end{array}$ \\
\hline $\begin{array}{l}\text { I'm not gonna be depending } \\
\text { on their fucking ass }\end{array}$ & Yo no voy a estar dependiendo de nadie. & $\begin{array}{c}\text { Depending on their fucking ass }< \\
\text { dependiendo de nadie } \varnothing\end{array}$ \\
\hline $\begin{array}{l}\text { A whole of motherfucking } \\
\text { white men }\end{array}$ & Muchos hombres blancos & $\begin{array}{c}\text { motherfucking white men }<\varnothing \\
\text { hombres blancos }\end{array}$ \\
\hline \multirow{2}{*}{$\begin{array}{l}\text { You cocksucking } \\
\text { motherfucker. Just fucking } \\
\text { let me out of here. I'll } \\
\text { fucking rip your fucking... } \\
\text { Stop the fucking car. Why } \\
\text { are you such a shit? }\end{array}$} & \multirow[b]{2}{*}{$\begin{array}{l}\text { Maldito hijo de perra. Déjame bajar del } \\
\text { auto. Por favor, déjame bajar del auto }\end{array}$} & Fucking $<\varnothing$ \\
\hline & & $\begin{array}{c}\text { Why are you such a shit }<\varnothing \text { (y adición } \\
\text { de: "por favor") }\end{array}$ \\
\hline Not a goddam thing & Absolutamente nada & Goddam $<\varnothing$ \\
\hline \multirow{2}{*}{$\begin{array}{l}\text { What the fuck do you care? } \\
\text { We're talking about fucking } \\
\text { per diem here }\end{array}$} & \multirow{2}{*}{$\begin{array}{c}\text { Qué importa. Hay que aprovechar la } \\
\text { oportunidad }\end{array}$} & What the fuck $<\varnothing$ \\
\hline & & Fucking $<\varnothing$ \\
\hline $\begin{array}{l}\text { Set crush? Nigga please, you } \\
\text { were his set wife }\end{array}$ & $\begin{array}{l}\text { ¿Romance de set? Por favor, era su } \\
\text { esposa en el set }\end{array}$ & Nigga please $<\varnothing$ por favor \\
\hline $\begin{array}{l}\text { Bitch, you two are still into } \\
\text { each other and you know it }\end{array}$ & $\begin{array}{l}\text { Querrás decir que se siguen gustando los } \\
\text { dos y lo sabes }\end{array}$ & Bitch $<\varnothing$ \\
\hline Your ass is just mad & Solo estás molesta & Ass $<\varnothing$ \\
\hline \multirow{2}{*}{$\begin{array}{c}\text { Bitch, you need to get over } \\
\text { that shit }\end{array}$} & \multirow{2}{*}{ Solo tienes que superarlo } & Bitch $<\varnothing$ \\
\hline & & To get over that shit $<$ superarlo $\varnothing$ \\
\hline \multirow{2}{*}{$\begin{array}{c}\text { Damm it! Shit! Here he } \\
\text { comes. Shit! Hold on Zoe. } \\
\text { Fuck you, motherfucker, fuck } \\
\text { you! }\end{array}$} & \multirow[b]{2}{*}{$\begin{array}{l}\text { ¡Rayos!, agárrate, por dios, agárrate. } \\
\text { Agárrate con fuerza, Zoe, cuidado }\end{array}$} & Shit y shit $<\varnothing$ y $\varnothing$ \\
\hline & & $\begin{array}{c}\text { Fuck you, motherfucker, fuck } \\
\text { you! }<\varnothing, \varnothing, \varnothing\end{array}$ \\
\hline $\begin{array}{c}\text { Redneck, lunatic bastard. Get } \\
\text { away from me }\end{array}$ & Agárrate, Zoe. Cuidado, Zoe. ¡Loco! & Redneck, lunatic bastard $<\varnothing$, loco $\varnothing$ \\
\hline \multirow{2}{*}{$\begin{array}{l}\text { You wanna get hot? Suck on } \\
\text { this for a while, bitch }\end{array}$} & \multirow{2}{*}{ Maldita bruja } & Get hot $<\varnothing$ \\
\hline & & Suck on $<\varnothing$ \\
\hline Get ready to fly, bitch & Ahora sí, prepárate para volar & Bitch $<\varnothing$ \\
\hline $\begin{array}{l}\text { He's right back on our } \\
\text { fucking ass }\end{array}$ & Va tras nosotras & Fucking ass $<\varnothing$ \\
\hline
\end{tabular}




\begin{tabular}{|c|c|c|}
\hline Versión original & Doblaje para Hispanoamérica & $\begin{array}{l}\text { Estrategia de atenuación: } \\
\text { CIRCUNLOOUIO }\end{array}$ \\
\hline $\begin{array}{l}\text { If you don't bust their balls } \\
\text { a little bit, they never gonna } \\
\text { respect you }\end{array}$ & $\begin{array}{l}\text { Si les das lo que quieren la primera } \\
\text { noche, nunca te respetarán }\end{array}$ & bust their balls $<$ dar lo que quieren \\
\hline Fuck that & Eso no & Fuck that $<$ Eso no \\
\hline $\begin{array}{l}\text { I think you got Mike laid } \\
\text { tonigh }\end{array}$ & $\begin{array}{l}\text { Creo que le conseguiste a Mike una } \\
\text { buena noche }\end{array}$ & $\begin{array}{c}\text { To get laid }<\text { conseguir una buena } \\
\text { noche }\end{array}$ \\
\hline $\begin{array}{l}\text { Hell yeah, the next job after } \\
\text { this one is definitely gonna } \\
\text { suck }\end{array}$ & $\begin{array}{l}\text { Claro que sí, el próximo trabajo va a } \\
\text { ser un horror }\end{array}$ & Hell yeah<claro que sí \\
\hline He's a stand-in fucker & Se acuesta con las dobles & Fucker $<$ se acuesta \\
\hline $\begin{array}{l}\text { You need to break that } \\
\text { nigga off a piece }\end{array}$ & Tienes que dejar hacer algo al tipo & $\begin{array}{c}\text { To break off a piece }<\text { dejarlo hacer } \\
\text { algo }\end{array}$ \\
\hline Get your ass up here & Te necesito en el asiento delantero & Get your ass up $<$ te necesito \\
\hline Oh, shit! & ¡Venga ya! & Shit $<$ ¡venga ya! \\
\hline Run away with your ass & Corre, maldito infeliz & $\begin{array}{l}\text { Run away with your ass }>\text { maldito } \\
\text { infeliz (Circunloquio y cambio de } \\
\text { significado) }\end{array}$ \\
\hline
\end{tabular}

\begin{tabular}{|c|c|c|}
\hline Versión original & Doblaje para Hispanoamérica & $\begin{array}{l}\text { Estrategia de atenuación: } \\
\text { EUFEMISMO Y TRASPLANTE }\end{array}$ \\
\hline \multirow{2}{*}{$\begin{array}{l}\text { I've gotta take the world's } \\
\text { biggest fucking pee }\end{array}$} & \multirow{2}{*}{ Tengo que ir al maldito baño } & Fucking $<$ maldito \\
\hline & & Pee $<$ baño \\
\hline $\begin{array}{l}\text { It's not my fucking job to } \\
\text { supply all of you with weed } \\
\text { everytime we go out }\end{array}$ & $\begin{array}{l}\text { No es mi maldito trabajo ser la } \\
\text { proveedora oficial de hierba }\end{array}$ & Fucking $<$ maldito \\
\hline $\begin{array}{l}\text { It's not about the money, } \\
\text { it's about the pain in the ass } \\
\text { of scoring }\end{array}$ & $\begin{array}{c}\text { No es por el dinero, es por la molestia } \\
\text { de conseguir hierba }\end{array}$ & Pain in the ass $<$ molestia \\
\hline $\begin{array}{l}\text { We made out on the couch } \\
\text { for twenty minutes }\end{array}$ & $\begin{array}{l}\text { Nos besamos en el sofá por veinte } \\
\text { minutos }\end{array}$ & made out $<$ besarse \\
\hline I was straddling him & Tenía mis piernas sobre él & Straddle $<$ tener las piernas sobre \\
\hline No hooking up tonigh & No pueden irse con nadie hoy & Hook up $<$ irse con alguien \\
\hline \multirow{2}{*}{$\begin{array}{l}\text { Horny boys trying to get } \\
\text { their fuck on with my } \\
\text { daugther }\end{array}$} & \multirow{2}{*}{$\begin{array}{l}\text { Una banda de libidinosos que quiere } \\
\text { acostarse con mi hija }\end{array}$} & Horny boys $<$ libidinosos \\
\hline & & To get their fuck on $<$ acostarse con \\
\hline $\begin{array}{l}\text { Ok. What the fuck are you } \\
\text { doing? }\end{array}$ & ¿Qué diablos estás haciendo? & What the fuck $<$ qué diablos \\
\hline \multirow{2}{*}{$\begin{array}{l}\text { Listen, double fucks. I'm } \\
\text { not gonna fuck him }\end{array}$} & \multirow{2}{*}{$\begin{array}{l}\text { Escuchen, idiotas. No voy a acostarme } \\
\text { con él }\end{array}$} & double fucks $<$ idiotas \\
\hline & & To fuck him<Acostarme con él \\
\hline $\begin{array}{l}\text { He got pretty banged up } \\
\text { himself }\end{array}$ & Él también terminó muy lastimado & To bang up $<$ lastimado \\
\hline Motherfuckers & Esquizofrénicas & $\begin{array}{l}\text { (Eufemismo con cambio de } \\
\text { significado) }\end{array}$ \\
\hline Kim doesn’t give a shit & A Kim no le interesa & give a shit $<$ interesar \\
\hline $\begin{array}{l}\text { Hell yeah, the next job after } \\
\text { this one is defnitely gonna } \\
\text { suck }\end{array}$ & $\begin{array}{c}\text { Claro que sí, el próximo trabajo va a ser } \\
\text { un horror }\end{array}$ & Is gonna suck<es un horror \\
\hline Dick department & Sección de penes & Dick $<$ pene (Trasplante) \\
\hline Were you two fucking? & ¿Ustedes se acostaban? & Fuck $<$ acostarse \\
\hline
\end{tabular}




\section{Continuación}

\begin{tabular}{|c|c|c|}
\hline Versión original & Doblaje para Hispanoamérica & $\begin{array}{l}\text { Dstrategia de atenuación: } \\
\text { EUFEMISMO Y TRA SPLANTE }\end{array}$ \\
\hline $\begin{array}{l}\text { You need to break that } \\
\text { nigga off a piece }\end{array}$ & Tienes que dejar hacer algo al tipo & Nigga $<$ tipo \\
\hline \multirow{2}{*}{$\begin{array}{l}\text { You're not fucking him, } \\
\text { you're not sucking him, } \\
\text { you're not giving him any } \\
\text { tongue }\end{array}$} & \multirow[b]{2}{*}{$\begin{array}{c}\text { No te acuestas con él, no le haces sexo } \\
\text { oral, no le das nada de lengua }\end{array}$} & Fucking $<$ acostarse \\
\hline & & $\begin{array}{c}\text { Sucking him }<\text { hacerle sexo oral } \\
\text { (Trasplante) }\end{array}$ \\
\hline $\begin{array}{l}\text { Fuck both of you and your } \\
\text { little high five }\end{array}$ & Váyanse al diablo con su burla & $\begin{array}{c}\text { Fuck both of you > váyanse al } \\
\text { diablo }\end{array}$ \\
\hline \multirow{2}{*}{$\begin{array}{l}\text { Before you can claim a } \\
\text { nigga, you've got to claim a } \\
\text { nigga and you could start } \\
\text { giving him a handjob }\end{array}$} & \multirow[b]{2}{*}{$\begin{array}{l}\text { Para domar a un tío, hay que tenerlo y } \\
\text { podrías empezar masturbándolo }\end{array}$} & Nigga $<$ tío \\
\hline & & Handjob $<$ masturbar (Trasplante) \\
\hline $\begin{array}{l}\text { You're starting to get dirty } \\
\text { on him, it blows their mind }\end{array}$ & $\begin{array}{c}\text { De pronto te pones sexi y los vuelves } \\
\text { tus esclavos }\end{array}$ & Dirty $<$ sexy \\
\hline Bullshit on that & Al diablo con eso & Bullshit $<$ diablo \\
\hline $\begin{array}{c}\text { Faster, you black bitch, } \\
\text { faster }\end{array}$ & Más rápido, negra inútil & $\begin{array}{c}\text { Bitch }<\text { inútil (Eufemismo y cambio } \\
\text { de significado) }\end{array}$ \\
\hline $\begin{array}{l}\text { Is that fast enough for your } \\
\text { bonny ass? }\end{array}$ & Así está bien para tu trasero huesudo? & Bonny ass $<$ trasero huesudo \\
\hline Damm it! & ¡Rayos! & Dammit $<$ rayos \\
\hline Sorry I call you black bitch & Perdona por llamarte negra inútil & $\begin{array}{c}\text { Bitch }<\text { inútil (Eufemismo y cambio } \\
\text { de significado) }\end{array}$ \\
\hline $\begin{array}{l}\text { I forgive you, hold the fuck } \\
\text { on. }\end{array}$ & Te perdono pero agárrate, ¡rayos! & Fuck $<$ rayos \\
\hline $\begin{array}{l}\text { Hey, hey, ladies. That was } \\
\text { fun! Well, adiós. Ah, fuck, } \\
\text { shit, fuck }\end{array}$ & $\begin{array}{l}\text { Oigan, oigan señoritas. Eso fue } \\
\text { divertido. Bueno, adiós. Ah, qué } \\
\text { demonios }\end{array}$ & Fuck, shit, fuck< $<$ qué demonios \\
\hline $\begin{array}{c}\text { You better run, } \\
\text { motherfucker }\end{array}$ & Será mejor que corras, maldito & Motherfucker $<$ maldito \\
\hline \multirow{2}{*}{$\begin{array}{l}\text { Oh, shit, damm it. You } \\
\text { fucking bitch }\end{array}$} & \multirow{2}{*}{ Rayos, es una bruja, odio que haga eso } & $\begin{array}{c}\text { Oh, shit, dammit }<\text { rayos } \\
\text { (Eufemismo) }\end{array}$ \\
\hline & & $\varnothing<$ odio que haga eso (Adición) \\
\hline
\end{tabular}

Una vez analizados los ejemplos expuestos, podemos destacar que el eufemismo se trata de la estrategia de atenuación más empleada en el corpus (supone más del $50 \%$ de ejemplos analizados). Como ejemplo, nos podemos referir a casos como "dirty", traducido como "sexy", o "pain in the ass", traducido por el más genérico "molestia".

Dentro de los casos de eufemismo (35 de 69), encontramos tres ejemplos de trasplante: "sucking him" como "hacerle sexo oral", "dick" por "pene" y "handjob" por "masturbar".

En algunos casos la sustitución del tabú por un eufemismo puede conllevar un cambio del significado con respecto al texto origen, como ha sucedido con "motherfucker", que ha sido traducido como "ezquizofrénica", "bitch", como "inútil" (en dos ocasiones), o la expresión "run away with your ass" por "maldito infeliz". 
Otra de las estrategias de atenuación que encontramos en el doblaje analizado es el circunloquio. De los 9 casos analizados podemos señalar los siguientes ejemplos: "bust their balls" como "darles lo que quieren", "to break off a piece" por "dejarle hacer algo" y "get your ass up here" como "te necesito aquí".

Por último, la estrategia de la omisión o elipsis es una de las más frecuentes del corpus, con 24 apariciones. Especialmente se omiten términos como "fucking", "fuck", "bitch", "suck", etc.

Con objeto de hacer más sencilla la lectura de los resultados, ofrecemos el siguiente gráfico:

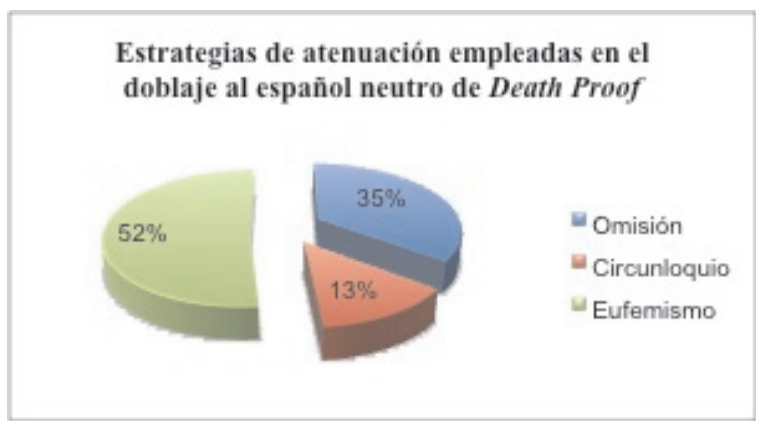

En relación a los términos del texto origen más afectados por la atenuación (plasmada en eufemismos y omisiones especialmente), podemos citar el término inglés "fuck" y sus derivados del tipo "fucking", "fucker"10, etc. Este término, que aparece en más de 19 ocasiones en los ejemplos analizados, ha sido traducido mediante el empleo de los siguientes eufemismos: "maldito" (dos casos), "acostarse" (tres casos), "diablos", "idiotas", "rayos" y "demonios". Sin embargo, lo más frecuente ha sido la omisión del disfemismo en el texto meta (ocho ocasiones).

También ha sido frecuente encontrar el término "shit", que suele ser omitido en el doblaje (en cuatro ocasiones). Lo podemos hallar traducido con los eufemismos "demonios" y "rayos" o para "she doesn't give a shit" mediante el circunloquio "no le interesa".

Nos gustaría destacar otro procedimiento que no podemos incluir precisamente en casos de eufemismo, circunloquio u omisión pero que nos ha resultado interesante. Se trata del caso de "Oh, shit, damm it. You fucking bitch", traducido como "Rayos, es una bruja, odio que haga eso", en el que observamos la adición de la última frase, probablemente para compensar el tono general.

Para finalizar, mencionaremos que las palabrotas y alusiones sexuales han sido las más afectadas por la atenuación llevada a cabo por el traductor. Esto ha dado como resultado una traducción en la que la presencia del lenguaje neutral es mucho mayor que en el texto original en inglés.

10 Analizado en todas sus apariciones como distintas categorías gramaticales. 


\section{Conclusiones}

Como hemos podido observar a lo largo del presente trabajo, los procedimientos lingüísticos más comunes empleados en el doblaje hispanoamericano de la película de Tarantino Death Proof (A prueba de muerte, 2007) para la atenuación del lenguaje soez son la omisión (en un $34,78 \%$ de los casos), el procedimiento léxico del eufemismo (presente en un 50, 72\% de ejemplos), así como el circunloquio o elipsis gramatical (empleado en un 13,04\% de casos). Por otra parte, el traductor ha hecho uso de las estrategias de atenuación en aquellos casos en los que el texto original presentaba un lenguaje cargado de referencias sexuales.

Pensamos que los doblajes hispanoamericanos como el que hemos analizado tienden al empleo de las estrategias de atenuación debido a que convergen dos tendencias. Por un lado, el hecho de que el español neutro sea la variedad lingüística utilizada en estos doblajes propicia que la lengua se desprenda de modismos, coloquialismos o vulgarismos, ya que este tipo de lenguaje suele estar marcado diatópicamente (y hemos de recordar que uno de los objetivos de esta variedad es la de ser válida para cualquier hablante hispanoamericano, así como la de no ser vinculada a una determinada zona geográfica). Por otro lado, el empleo de esta variedad lingüística condiciona las expectativas de los espectadores, habituados a escuchar en estos doblajes un lenguaje plano, neutro. Es por ello por lo que el lenguaje audiovisual, sea por esta causa o por otra, es más propenso que otros tipos de lenguaje al empleo de estrategias de atenuación.

\section{Referencias bibliográficas}

Abellán, M., Censura y creación literaria en España (1939-1976). Barcelona: Península 1980. AixelÁ Franco, J. / ABIo Villarig, C., «Manipulación ideológica y traducción: atenuación e intensificación moral en la traducción de la novela negra norteamericana al español (1933-2001)», Hermeneus 1, 11 (2009), 109-144.

AL-QuinaI, J., «Manipulation and censorship in translated texts», en: Romano GarCíA, M. L. (ed.), II AIETI. Actas de II Congreso Internacional de la Asociación Ibérica de Estudios de Traducción e Interpretación. Madrid: AIETI 2005, 488-525.

Del Teso Martín, E., «Cambio semántico, impropiedad y eufemismo», Verba 15 (1988), 183-204.

García Aguiar, L. C. / García JimÉNEZ, R., «Análisis descriptivo de los rasgos lingüísticos del español neutro en el doblaje de la serie televisiva The Flintstones (Los Picapiedra)», Interlingüistica XXI (2010), 1049-1058.

GarCía AgUiar, L. C. / GarCía JimÉNEZ, R., «La influencia del sistema meta en traducción: el doblaje de Los Picapiedra al español neutro», Estudios de Traducción 1 (2011), 127-138.

García Aguiar, L. C. / García Jiménez, R., «Análisis descriptivo de dos traducciones al español: Death Proof en español latino y en español de España», Interlingüistica XXII (2012), 407-417.

GómEZ CASTRO, C., «Traducción y censura de textos narrativos durante la España franquista: algunas prácticas traductoras», ponencia presentada en el V Congreso AIETI: Actualidad e investigación en los estudios de Traducción e Interpretación, celebrado en Castelón los días 16-18 de febrero de 2011. 
Holmes, J. S., «The Name and Nature of Translation Studies», en: Holmes, J. S. (ed.), Translated! Papers on Literary Translation and Translation Studies. Ámsterdam: Rodopi $1988,67-80$.

Miquel CorTÉs, C., «Traducción y (auto)censura: el caso de Kill Bill en España y Lationamérica», en Jornades de Foment de la Investigació, Castellón: Universitat Jaume I (2005), 1-19. Disponible electrónicamente en < $<$ http://www.uji.es/bin/publ/edicions/ jfi10/trad/1.pdf $>$ [Última consulta: 04-XII-2012].

Montes Giraldo, J. J., «La atenuación frente al eufemismo», Boletín de la Academia Colombiana 54, 219-220 (2003), 67-70.

PAJARES GimÉNEZ, M. T., «El español "neutro” y la oralidad», en: SÁEZ, D. et alii (eds.), Últimas tendencias en traducción e interpretación. Madrid: Iberoamericana-Vervuert 2011, 45-58.

Pons Rodríguez, L., «Español de España y español de América en el doblaje. La variación lingüística a través de un estudio de caso», en: SÁEZ, D. et alii (eds.), Últimas tendencias en traducción e interpretación. Madrid: Iberoamericana-Vervuert 2011, 59-76.

RABADÁN, R., «Tendencias teóricas en los estudios contemporáneos de traducción», en: FERNÁNDEZ Nistal, P. (ed.), Estudios de Traducción. Valladolid: Universidad de Valladolid 1992, 45-50.

RAMíREZ LuENGO, J. L., «Imaginar lo imposible: algunas reflexiones sobre el denominado español neutro», en: SÁEz, D. et al. (eds.), Últimas tendencias en traducción e interpretación. Madrid: Iberoamericana-Vervuert 2011, 17-26.

ScandurA, G. L., «Sex, Lies and TV: Censorship and Subtitling», Meta XLIX, 1 (2004), 125-134.

Senabre SEMPERe, R., «El eufemismo como fenómeno lingüístico», Boletín de la Real Academia Española 51 (1971), 175-189.

Tarantino, Q., Grindhouse: Death Proof. Estados Unidos: Dimension Films 2007.

Tarantino, Q., A prueba de muerte. México: Pachamama Cine 2007.

Toledano Buendía, C., La traducción de la obscenidad. Santa Cruz de Tenerife: La Página Ediciones 2003.

Toury, G. In Seacrh of a Theory of Translation. Tel-Aviv: Porter Institute for Poetics and Semiotics, Tel Aviv University 1980.

Toury, G., Descriptive Studies and Beyond. Ámsterdam-Philadelphia: John Benjamins Publishings 1995. 